\title{
Ascorbic acid-catalyzed degradation of cyanidin-3-O- $\beta$-glucoside: Proposed mechanism and identification of a novel hydroxylated product
}

\author{
Nathan B. Stebbins ${ }^{\mathrm{a}}$, Luke R. Howard ${ }^{\mathrm{a}, *}$, Ronald L. Prior ${ }^{\mathrm{a}}$, Cindi Brownmiller ${ }^{\mathrm{a}}$, Rohana Liyanage ${ }^{\mathrm{b}}$, \\ Jackson O. Lay ${ }^{\mathrm{b}}$, Xiaoyu Yang ${ }^{\mathrm{c}}$ and Steven Y. Qian ${ }^{\mathrm{c}}$ \\ ${ }^{a}$ Department of Food Science, University of Arkansas, Fayetteville, AR, USA \\ ${ }^{\mathrm{b}}$ University of Arkansas Statewide Mass Spectrometry Facility, Fayetteville, AR, USA \\ ${ }^{\mathrm{c}}$ Department of Pharmaceutical Science, North Dakota State University, Fargo, ND, USA
}

Received 25 November 2015; accepted 2 April 2016

\begin{abstract}
.
BACKGROUND: Many brightly colored fruits and vegetables owe their pigmentation and beneficial health effects to anthocyanins. Unfortunately, anthocyanins in the presence of ascorbic acid are readily degraded over juice processing and storage, which adversely affects color stability and potential health benefits.

OBJECTIVE: This project focused on the effect of ascorbic acid as a catalyst in anthocyanin degradation.

METHODS: The project involved searching for novel pigmented compounds in a simple model system composed of the most common anthocyanin cyanidin-3-O- $\beta$-glucoside and ascorbic acid, and a second model system consisting of blackberry extract supplemented with ascorbic acid. Degradation products were identified by HPLC-PDA and HPLC-MS. ESR was used to monitor hydroxyl radical formation in the model systems.

RESULTS: Over 72 hours at ambient temperature, $67 \%$ of cyanidin-3- $O$ - $\beta$-glucoside was lost in the model system during which time an unknown pigmented compound was formed. The unknown compound was also formed in a more complex model system consisting of blackberry extract and ascorbic acid. HPLC with PDA monitoring at $510 \mathrm{~nm}$ was used to detect a novel compound and HPLC-ESI-MS 3 allowed a proposed structure to be built based on the fragmentation patterns. The unknown structure formed via oxidation of cyanidin 3- $O$ - $\beta$-glucoside by ascorbic acid was identified as 6-hydroxy-cyanidin3-O- $\beta$-glucoside. The mechanism was substantiated with malvidin-3- $O$ - $\beta$-glucoside and ascorbic acid, which produced a hydroxylated malvidin-3-O- $\beta$ - glucoside. Production of hydroxyl radical in the base and blackberry model systems was confirmed by ESR.

CONCLUSIONS: We propose that the pigmented compound is formed from hydroxyl radicals via the Haber-Weiss reaction. The addition of food grade hydroxyl radical scavengers to juices may be a viable treatment to prevent ascorbic acid-catalyzed degradation of anthocyanins.
\end{abstract}

Keywords: Anthocyanin, ascorbic acid, blackberry, cyanidin-3-O- $\beta$-glucoside, degradation product, hydroxyl radical, tandem mass spectrometry

\footnotetext{
${ }^{*}$ Corresponding author: Dr. Luke Howard, Department of Food Science, University of Arkansas, 2650 N. Young Avenue, Fayetteville, AR 72704, USA. Tel.: +1 479575 2978; Fax: +1 479575 6936; E-mail: lukeh@uark.edu. 


\section{Introduction}

The vibrant blue, red, and purple colors of berries and many other fruits are due to anthocyanins. In addition to serving as pigments, anthocyanins are thought to afford protection against chronic diseases through antioxidant, anti-inflammatory, and anti-cancer activities [1]. Fresh berry consumption is limited by seasonal availability and short shelf life. Therefore, many berries are processed and consumed in shelf stable products such as juices, jellies, and jams. Unfortunately, anthocyanins are susceptible to degradation during berry processing and storage of processed products at ambient temperature [2]. Anthocyanin losses have been attributed to many factors including exposure to heat, oxygen, enzymes, metals, and ascorbic acid [3-6]. Oxidation by secondary oxidizing agents, such as hydroxyl radicals and superoxide, are thought to be contributing factors to color deterioration [7].

Many studies report that anthocyanins and the color of processed berry products are unstable in the presence of ascorbic acid [8-11]. Two different mechanisms have been proposed for ascorbic acid (Asc) catalyzed degradation of anthocyanins. The first involves a condensation reaction between anthocyanins and other components, such as ascorbic or organic acids [3,8], and the second involves a radical mechanism leading to cleavage of the anthocyanin into phenolic acids $[11,12]$. Much of the research related to structural changes in anthocyanins has been conducted on wine. Pyranoanthocyanins, which are anthocyanin conjugates with an additional pyran ring between $\mathrm{C}-4$ and the 5-OH have been identified in several studies [13-15]. Pyranoanthocyanins can be formed from reactions between anthocyanins and fermentation products, such as acetaldehyde, pyruvic and acetoacetic acids $[13,16]$, as well as hydroxycinnamic acids $[17,18]$. These compounds, which are consistent with the condensation product hypothesis proposed by Jurd [8] appear more $\mathrm{pH}$ stable [19] and have been detected in wine stored for several years [20]. Other than pyranoanthocyanins, polymeric pigments are a potential source of long-term color stability [21]. Polymeric pigments are the condensation product of anthocyanins and flavanols [22] and most often detected by MALDI-TOF-MS [23-25].

The fate of anthocyanins in the presence of Asc is unclear. This study was undertaken to identify novel degradation products of anthocyanins formed via Asc mediated oxidation using a cyanidin-3-O- $\beta$-glucoside (C3G) model system and blackberry model system.

\section{Materials and methods}

\subsection{Materials}

Cyanidin-3-O- $\beta$-glucoside standard ( $>95 \%)$ was purchased from Chromadex (Irvine, CA, USA). Malvidin3-O- $\beta$-glucoside standard ( $>95 \%$ ) was purchased from Extrasynthese (Genay, France). HPLC grade methanol was purchased from EMD Millipore (Billerica, MA, USA) and formic acid from Fischer-Scientific (Fair Lawn, NJ, USA). ACS grade L-ascorbic acid was obtained from VWR International (Radnor, PA, USA), Dimethyl sulfoxide (DMSO) was obtained from Sigma-Aldrich (St. Louis, MO, USA). High-purity $\alpha$-(4-pyridyl-1-oxide)N-tert-butylnitrone (POBN) was purchased from Alexis Biochemicals (San Diego, CA, USA). The University of Arkansas Horticulture department provided frozen blackberries. Alstroemeria flowers were purchased from a local florist.

\subsection{Formulation of model systems}

\subsubsection{Formulation of base model system and blackberry model system for HPLC and HPLC/MS analyses}

The base model system contained C3G $(200 \mu \mathrm{g} / \mathrm{mL})$ and ascorbic acid $(320 \mu \mathrm{g} / \mathrm{mL})$ dissolved in water acidified to $\mathrm{pH} 3.6$ by $\mathrm{HCl}$. Each $4 \mathrm{~mL}$ replicate was injected once every 70 minutes based on the HPLC gradient duration over 72 hours. The aqueous blackberry extract was concentrated to $200 \mu \mathrm{g} / \mathrm{mL} \mathrm{C} 3 \mathrm{G}$ and supplemented to contain $320 \mu \mathrm{g} / \mathrm{mL}$ Asc forming the blackberry model system. A model system containing malvidin-3-O- $\beta$-glucoside 
(M3G) $(200 \mu \mathrm{g} / \mathrm{mL})$ and Asc $(320 \mu \mathrm{g} / \mathrm{mL})$ dissolved in water acidified to $\mathrm{pH} 3.6$ by $\mathrm{HCl}$ was used to validate the process. Duplicates of each system were performed.

\subsubsection{Formulation of base model system and blackberry model system for ESR analysis}

$500 \mu \mathrm{L}$ of $200 \mu \mathrm{g} / \mathrm{mL}$ blackberry extract solution (or $200 \mu \mathrm{g} / \mathrm{mL}$ C3G solution) was mixed with $5 \mu \mathrm{L}$ of Asc stock solution $(32 \mathrm{mg} / \mathrm{mL})$ to generate $320 \mu \mathrm{g} / \mathrm{mL}$ final concentration in the sample. In order to trap free radicals generated from the system, $50 \mathrm{mM}$ POBN and $10 \mathrm{mM}$ DMSO was added to the sample. The system was maintained at room temperature. After mixing the Asc, DMSO, POBN and blackberry extract (or C3G), the samples were injected into the ESR instrument for analysis.

\subsection{Anthocyanin analysis}

\subsubsection{Blackberry extraction}

Frozen blackberries $(18.74 \mathrm{~g})$ were thawed at room temperature and triple extracted with $20 \mathrm{~mL}$ methanol/water/formic acid $(60: 37: 3 \mathrm{v} / \mathrm{v} / \mathrm{v})$, followed by $20 \mathrm{~mL}$ acetone/water/acetic acid $(70: 29.5: 0.5 \mathrm{v} / \mathrm{v} / \mathrm{v})$ using an IKA-T18 Ultra-Turrax (Wilmington, NC, USA) [26]. After filtering through Miracloth (CalBiochem, La Jolla, CA), the combined filtrates were evaporated to dryness in a Speed Vac Plus SC210A (Cambridge Scientific, Cambridge, MA) and brought up into $1 \mathrm{~mL}$ acidified water (pH 3.6 via $\mathrm{HCl}$ ).

\subsubsection{Alstroemeria extraction}

Dark red Alstroemeria incaica $\mathrm{v}$. Veronica flowers $(11 \mathrm{~g})$ were extracted with $50 \mathrm{~mL}$ methanol/water/formic acid $(60: 37: 3)$ using an IKA-T18 Ultra-Turrax (Wilmington, NC, USA). Samples were injected without further concentration for qualitative analysis and used the following HPLC-PDA and HPLC-ESI-MS ${ }^{3}$ methodology.

\subsubsection{HPLC-PDA analysis of anthocyanins and degradation products}

Chromatographic analyses were performed using dual Waters 515 pumps, 717plus autosampler, and a Waters 996 photodiode array detector (Milford, MA, USA). A Waters Symmetry $\mathrm{C}_{18}$ column $(4.6 \times 250 \mathrm{~mm} ; 5 \mu \mathrm{m})$ separated the analytes using a $1.2 \mathrm{~mL} / \mathrm{min}$ flow rate with $5 \%$ formic acid in water (Solvent A) and methanol (Solvent B). The elution began with 5\% B and rose to $15 \% \mathrm{~B}$ in $10 \mathrm{~min}$, from $15 \%$ to $35 \% \mathrm{~B}$ in $35 \mathrm{~min}$, from $35 \%$ to $100 \% \mathrm{~B}$ in $5 \mathrm{~min}$, followed by an isocratic wash for $10 \mathrm{~min}$ at $100 \% \mathrm{~B}$, then re-equilibration of the column with $5 \%$ B for $10 \mathrm{~min}$. UV-visible spectra were monitored from $200-700 \mathrm{~nm}$ and peak areas were recorded at $510 \mathrm{~nm}$.

\subsubsection{HPLC-electrospray ionization tandem mass spectrometry (LC-ESI-MSn) analysis of degradation products}

LC-ESI-MS ${ }^{\mathrm{n}}$ analysis was conducted using an HP 1000 series HPLC and a Bruker Esquire 2000 quadrapole ion trap mass spectrometer. Samples from both model system and blackberry extracts were separated using a Waters Symmetry $(4.6 \times 250 \mathrm{~mm} ; 5 \mu \mathrm{m})$ column and gradient as described above. The mass spectrometry analysis was performed in both positive and negative ion modes under the following conditions: capillary voltage at $4 \mathrm{kV}$ with polarity [+] for negative ion mode and [-] for positive ion mode analysis, nebulizer gas pressure 32 psi, dry gas flow $12 \mathrm{~L} / \mathrm{min}$, and skim voltage at $53.7 \mathrm{~V}$ and again polarity switched for respective mode. Ions were isolated and fragmented in quadrapole ion trap with excitation amplitude of 1.2 volts.

\subsubsection{Electron spin resonance (ESR) analysis}

Electron spin resonance analyses were operated on a Bruker Elexsys spectrometer at $9.76 \mathrm{GHz}$ and room temperature. ESR settings were modulation frequency, $100 \mathrm{kHz}$; modulation amplitude, $1.0 \mathrm{G}$; microwave power, $20 \mathrm{~mW}$; receiver gain, $2.5 \times 10^{5}$; sweep width, $70 \mathrm{G}$; and time constant, $0.65 \mathrm{~s}$. 


\subsubsection{Color monitoring of model systems}

The lightness (L), chroma (C), and hue (h) were measured on a Konica Minolta Chroma meter CR-400 and data processor DP-400 using the $\mathrm{L}^{*} \mathrm{C}^{*} \mathrm{~h}$ color scheme. (Konica Minolta, Japan). Measurements were taken every 2 hours during 12 diurnal hours, with 12 nocturnal hours without readings, over 72 hours. Four different samples were monitored: $\mathrm{C} 3 \mathrm{G}$ control, $\mathrm{C} 3 \mathrm{G}$ with Asc, blackberry extract control, and blackberry extract with Asc.

\section{Results and discussion}

\subsection{Unknown formation in base and blackberry model systems}

C3G was selected for study in the model system since it is the most common anthocyanin in blackberries [27] and in nature [28]. The ascorbic acid:anthocyanin ratio used in the model system is similar to that found in strawberries $[29,30]$.

To search for potential degradation products of anthocyanins, the base model system containing C3G and Asc was scanned for emerging peaks at $510 \mathrm{~nm}$. One salient peak appeared to increase as soon as one hour after addition of Asc to the base model system. The unknown peak continued to increase in area for approximately 30 hours before quickly falling to near zero area under the curve by 72 hours. Compound 1 had a $\lambda_{\max }$ of $500.6 \mathrm{~nm}$; therefore Fig. 1 displays the PDA signals of Compound 1 and C3G at $500 \mathrm{~nm}$ at $30 \mathrm{hrs}$. Compound 1 demonstrated greater hydrophilicity than $\mathrm{C} 3 \mathrm{G}$ eluting about 10 minutes earlier than C3G. The same peak in the blackberry system also had an earlier retention time and lower $\lambda_{\max }$ than $\mathrm{C} 3 \mathrm{G}$, but had nearly a 10 -fold drop in peak area (Fig. 2). The greater number of polyphenolic, radical-scavenging analytes in the blackberry system likely quenched the reaction mechanism resulting in protracted formation and lower production of Compound 1.

The dramatic effect of Asc on the degradation of C3G is featured in Fig. 3. In the C3G control, the peak area remains nearly constant over 70 hours, while the addition of Asc resulted in a rapid decrease in C3G peak area. This agrees with the findings of other researchers that anthocyanins are readily degraded in the presence of Asc $[10,11]$.

The Asc had a minimal affect on lightness (L) and chroma (C) values signifying no change in lightness or saturation, respectively (data not shown). Hue (h) angle increased from 20.3 to 29.7 immediately after addition of Asc indicating a shift from red to a more orange color. Hue values changed very little over the time course of the model system, thus the formation of the unknown compound had little to no effect on color. The L, C,

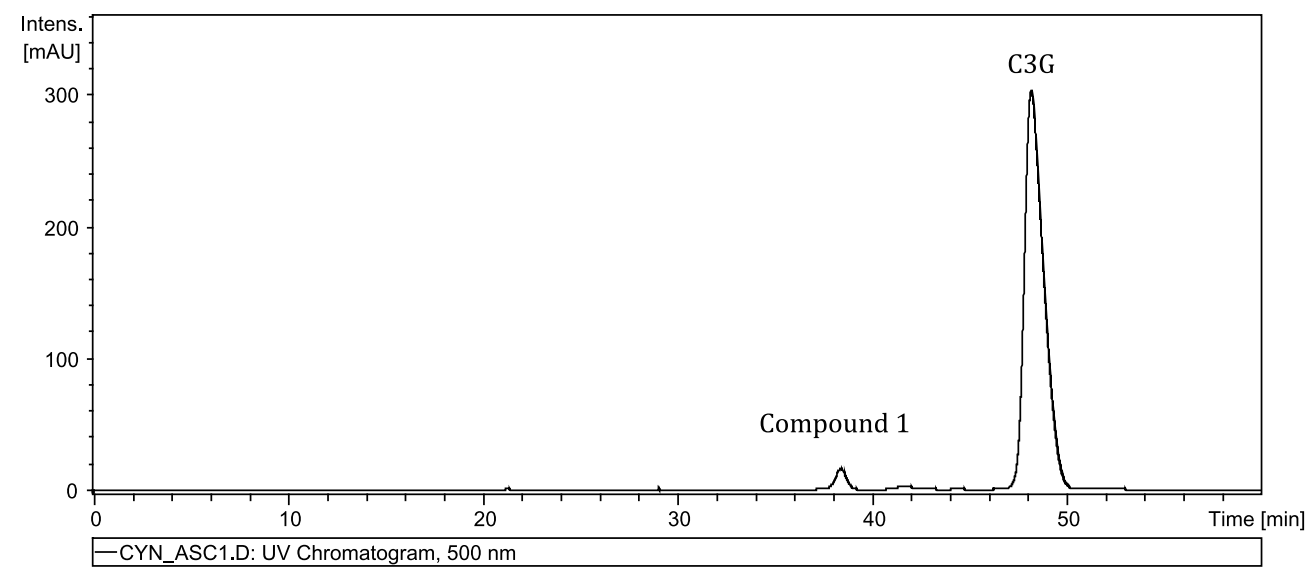

Fig. 1. HPLC chromatogram $(500 \mathrm{~nm})$ of Compound 1 and $\mathrm{C} 3 \mathrm{G}$ at 30 hours in the base model system. 


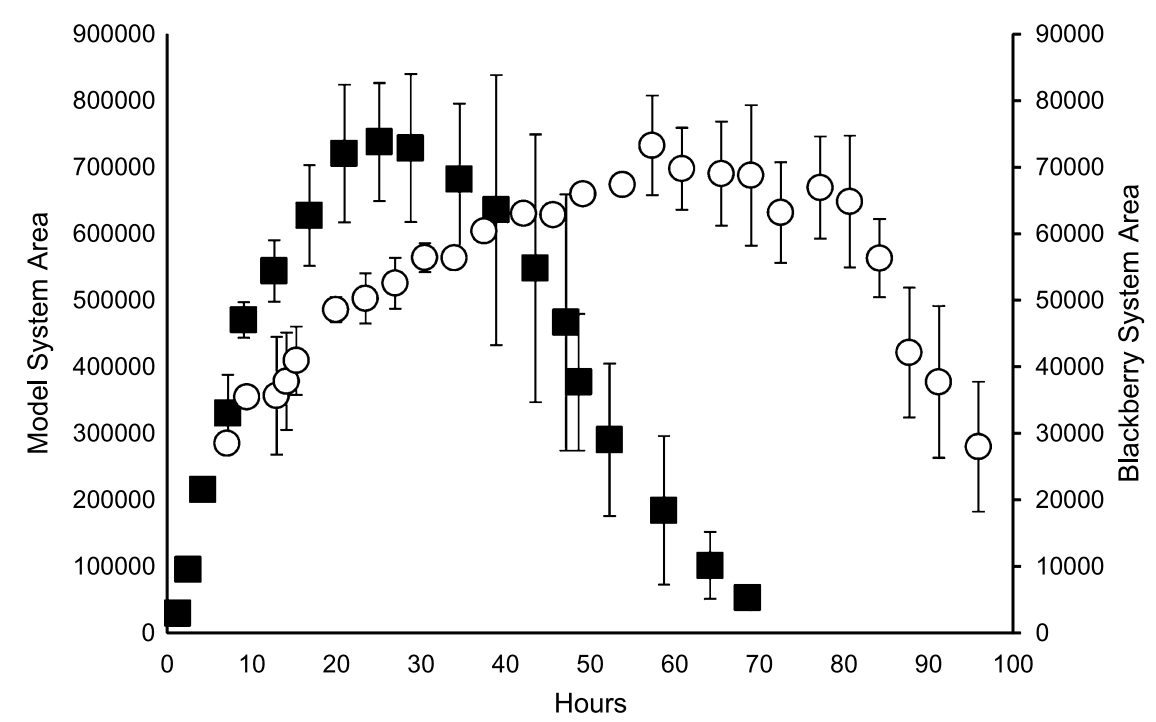

Fig. 2. Time course of Compound 1 formation in base ( $\square$ ) and blackberry $(\mathrm{O})$ model systems. Bars represent standard error $(n=2)$.

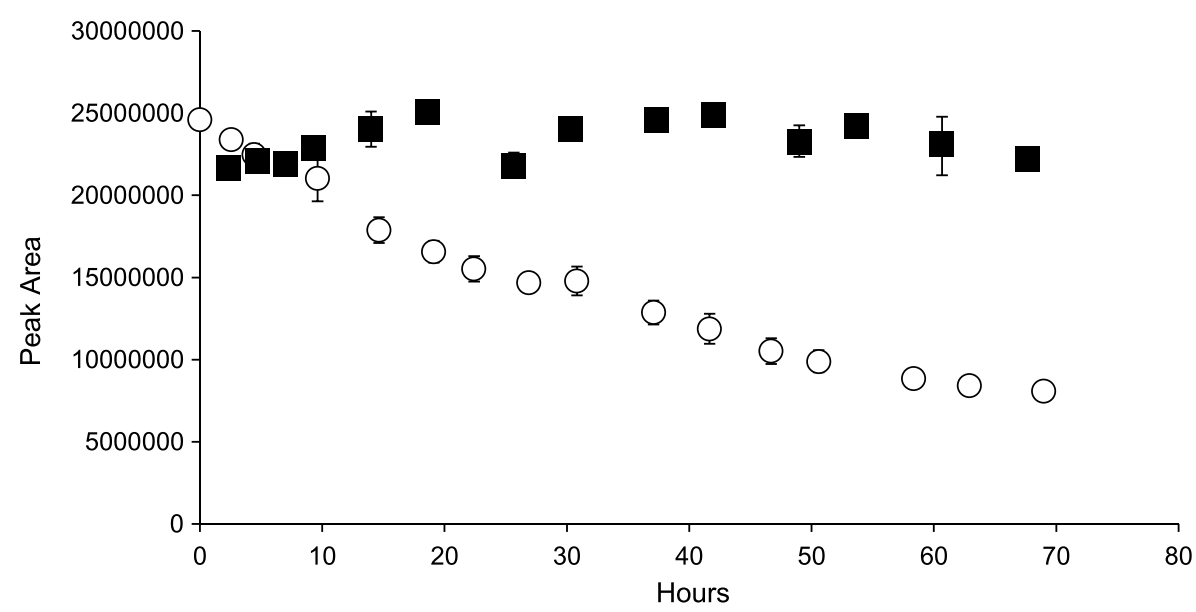

Fig. 3. Time course of $\mathrm{C} 3 \mathrm{G}$ control $(\mathbf{\square})$ and $\mathrm{C} 3 \mathrm{G}$ in base model system with ascorbic acid $(\mathrm{O})$. Bars represent standard error $(n=2)$.

and $\mathrm{h}$ values did not change much in the blackberry model systems, with and without Asc addition, over the course of 72 hours. The mechanism responsible for the immediate color change is not clear; it could be caused by copigmentation between the anthocyanin and Asc. Further research needs to be performed to answer this question.

\subsection{Structural elucidation of compound 1}

Tandem mass spectrometry yielded beneficial data toward structural elucidation, as seen in Fig. 4. Electrospray and tandem mass spectrometry are excellent tools for anthocyanin identification [31]. Comparing spectra of C3G and Compound 1, it was clear that Compound 1 had an $\mathrm{m} / \mathrm{z}$ value 16 amu greater than C3G. We suspect Compound 


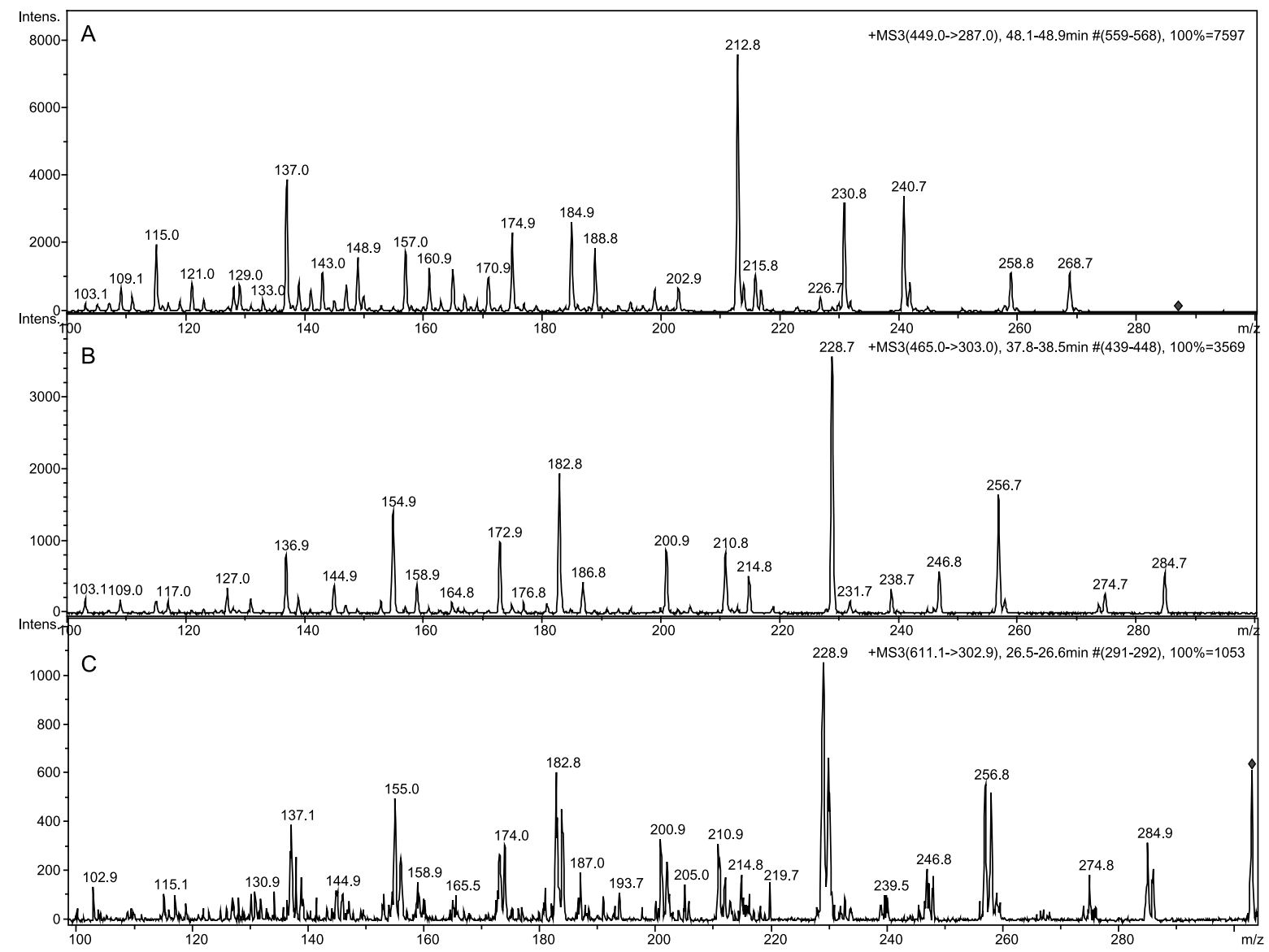

Fig. 4. LC-ESI-MS ${ }^{3}$ analysis of C3G (A), Compound 1 (B), and 6-hydroxycyanidin (C).<smiles>O=c1oc(-c2ccc(O)c(O)c2)cc2c(O)cc(O)cc12</smiles><smiles></smiles>

Fig. 5. Cyanidin-3-O- $\beta$-glucoside and proposed structure of Compound 1. 
1 to contain an additional hydroxyl group on one of the unsubstituted carbons. Compound 1 eluted earlier than C3G, which demonstrates increased hydrophilicity, strengthening the argument for an additional hydroxyl group. Anthocyanins often form polymers with a $\mathrm{C} 4 \Rightarrow \mathrm{C} 8$ linkage, but sometimes feature a $\mathrm{C} 4 \Rightarrow \mathrm{C} 6$ [32]. The dearth of reactivity at $\mathrm{C} 6$ in polymers is likely due to steric hindrance, which is not an issue in the hydroxyl addition. A related compound is 6-hydroxy-cyanidin-3-rutinoside, which is found in Alstroemeria flowers and described as less stable than cyanidin [33]. Both the rutinoside and glucoside are found in Alstroemeria flowers, but the rutinoside is found in much greater quantities [34]. An extract of dark red Alstroemeria flowers was analyzed with LC-ESI-MS ${ }^{\mathrm{n}}$ and compared to the ESI-MS ${ }^{3}$ of Compound 1 (Fig. 4). Although Alstroemeria contains a rutinoside, the sugar is fragmented by tandem mass spectrometry allowing a direct comparison of the aglycone: 6 -hydroxycyanidin to Compound 1 . Therefore we propose that Compound 1 is 6-hydroxy-cyanidin-3-O- $\beta$ glucoside (Fig. 5).

\subsection{Proposed mechanism responsible for the formation of compound 1}

Ascorbic acid can have varied effects on anthocyanin content. It has been reported that Asc can reduce enzymatic degradation of anthocyanins in grape juice [35]. Alternatively, Asc could produce hydrogen peroxide that would bleach anthocyanins [36-38]. Regardless of the presence of oxygen or nitrogen, Asc had a significant effect on anthocyanin degradation [3]. This finding led to an agreement with Jurd [8] that a condensation reaction occurred between the anthocyanin and Asc, which broke the conjugation and resulted in a loss of color. However, Jurd's condensation reaction mechanism includes a pentavalent carbon in the final product. The current research did not detect a condensation product between Asc and C3G while monitoring at 280 and $510 \mathrm{~nm}$. The mass spectrometry data confirms that no condensation products were detected. In the absence of Asc, a nitrogen flush had a small protective effect on anthocyanin retention compared to an oxygen rich environment [3], demonstrating a greater effect of Asc than oxygen.

(I) $\mathrm{Fe}^{3+}+\mathrm{O}_{2} \cdot-\rightleftharpoons \mathrm{Fe}^{2+}+\mathrm{O}_{2}$

(II) $2 \mathrm{O}_{2}^{--}+2 \mathrm{H}^{+} \rightleftharpoons \mathrm{H}_{2} \mathrm{O}_{2}+\mathrm{O}_{2}$

(III) $\mathrm{Fe}^{2+}+\mathrm{H}_{2} \mathrm{O}_{2} \rightleftharpoons \mathrm{OH}+{ }^{-} \mathrm{OH}+\mathrm{Fe}^{3+}$

Hydrogen peroxide formed via the Haber-Weiss reaction (Equations 1-3) [39] of Asc and iron or copper is the likely catalyst in forming Compound 1 from C3G [40]. There is very little iron and copper in the water used in the model system, but it may be enough to initiate the Haber-Weiss reaction. Although iron is a more efficient catalyst than copper [37], the model system contains more copper. Martell found that extremely low levels $(0.7 \mu \mathrm{M})$ of copper still exhibited catalytic activity and led to hydroxyl radical production [41]. The water used in the model and blackberry systems contained $0.2 \mu \mathrm{M}$ copper, which is a comparable concentration. Several researchers have studied the degradation kinetics of anthocyanin by $\mathrm{H}_{2} \mathrm{O}_{2}$ [42-45], but none reported the presence of Compound 1. Additionally, Tsuda et al. used a strong radical initiator in a model system with $\mathrm{C} 3 \mathrm{G}$, which resulted in the formation of protocatechuic acid and 4,6-dihydroxy-2-O- $\beta$-glucosyl-3-oxo-2,3-dihydrobenzofuran [46]. However, neither of these compounds were detected at $280 \mathrm{~nm}$ in our model systems.

Figure 6 depicts our proposed mechanism of formation for Compound 1 involving the hydroxyl radical from hydrogen peroxide. Since Compound 1's $\lambda_{\max }$ is $500.6 \mathrm{~nm}$, it must retain conjugation throughout the $\pi$ system and the mechanism must agree with the preservation of conjugation. The hydroxyl radical likely attacks C- 6 because that follows an ortho-addition between two hydroxyl groups on C-5 and C-7. Hydroxyl radical ortho-additions had the highest rate constants and lowest activation energy compared to meta- and para-additions to aromatic compounds [47]. There is potential for the hydroxyl group to add to C-8, but we do not see any peak separation or splitting via HPLC indicating a majority, if not the entire reaction proceeds to C-6 or the two positional isomers elute simultaneously. The instability of Compound 1 could be caused by the increase in $o$-quinone formation since the additional hydroxyl group allows $o$-quinone formation with either C-5 \& C-6 or C-6 \& C-7. Given the similarity in structure between $\mathrm{C} 3 \mathrm{G}$ and Compound 1, their molar absorptivity constants would be similar 


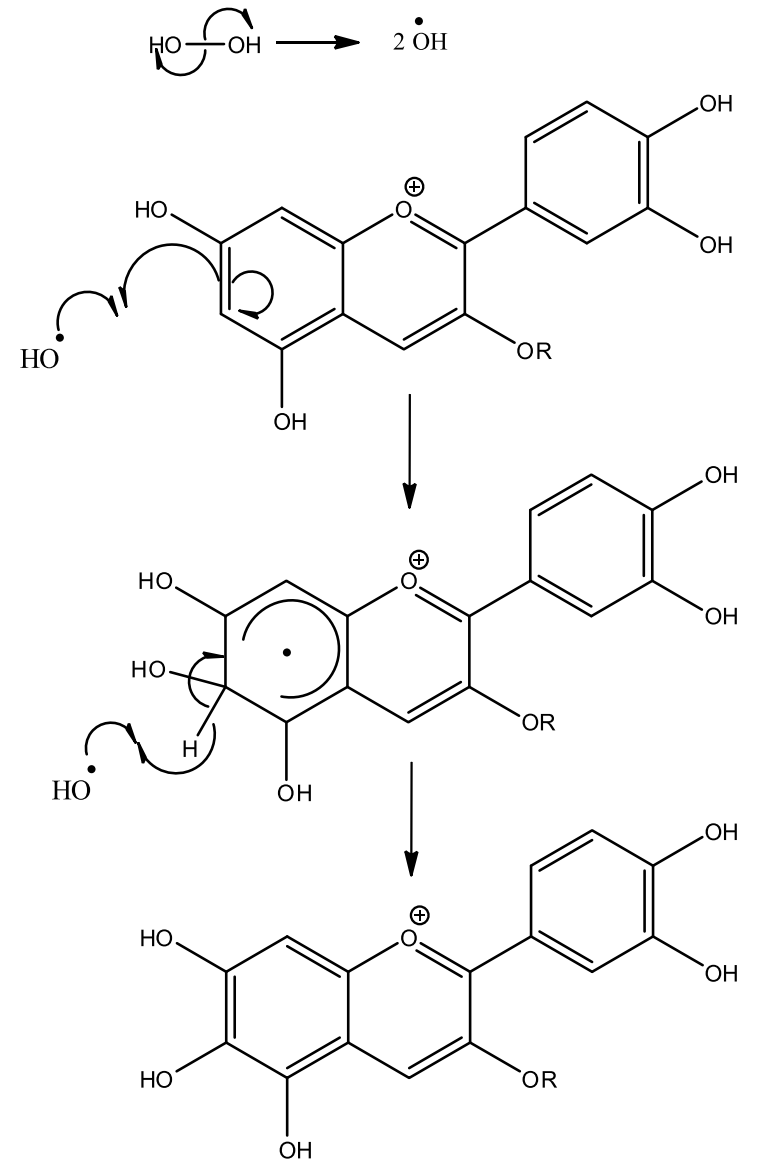

Fig. 6. Proposed mechanism of formation of Compound 1.

and allow comparison of their peak areas. In the model system, the area at Compound 1's $\mathrm{C}_{\max }$ is $7.56 \pm 0.53 \%$ of the area of C3G's degradation at that time. The blackberry system had even less of a conversion of C3G to Compound 1 with $1.03 \pm 0.22 \%$ of the area converted. The low transformation rate is indicative of a radical mechanism. The fate of the remaining percentage of $\mathrm{C} 3 \mathrm{G}$ is unknown at this time. As mentioned, no new peaks appeared while monitoring at neither 280 nor $510 \mathrm{~nm}$. The fate of anthocyanin degradation should continue to be studied.

\subsection{Confirmation of hydroxyl radical in the formation of compound 1}

Electron spin resonance (ESR) using POBN as a spin trapping agent was used to confirm the presence of hydroxyl radical in the base model system and blackberry model system supplemented with Asc. ESR can detect POBN spin adduct of methyl radical $\left(\mathrm{CH}_{3}\right)$ that is generated from the reaction of DMSO and $\mathrm{HO}^{\prime}$ as the following reaction (DMSO+HO $\longrightarrow \mathrm{CH}_{3} \mathrm{SO}_{2} \mathrm{H}+\mathrm{CH}_{3}$ ) [47-50]. In Fig. 7, no ESR spectra were observed for 1) blackberry extract + DMSO + POBN, 2) blackberry extract + Asc + DMSO, 3) blackberry extract+Asc+POBN, or 4) Asc + DMSO + POBN. However, the ESR spectrum we see in 5) blackberry 
A) $B E+D M S O+P O B N$

B) $B E+A s c+D M S O$

C ) $B E+A s c+P O B N$

D ) Asc+DMSO+POBN

E ) $B E+A s C+D M S O+P O B N$

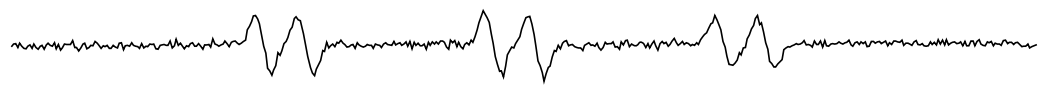

F) $\mathrm{C} 3 \mathrm{G}+\mathrm{AsC}+\mathrm{DMSO}+\mathrm{POBN}$

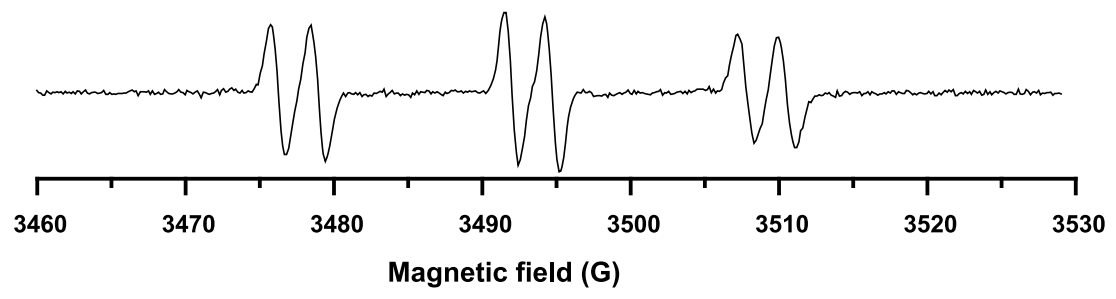

Fig. 7. ESR spectra of POBN spin trapped radical adduct in blackberry model system. The reaction mixture contained $200 \mu \mathrm{g} / \mathrm{mL}$ blackberry extract (BE) solution (or $200 \mu \mathrm{g} / \mathrm{mL}$ C3G solution), $320 \mu \mathrm{g} / \mathrm{mL}$ ascorbic acid, $50 \mathrm{mM}$ POBN, and $10 \mathrm{mM}$ DMSO. ESR spectrum of the reaction excluding: (A) ascorbic acid, (B) POBN, (C) DMSO, (D) blackberry extract, and (E-F) ESR spectra of the complete reaction system with blackberry and with pure $\mathrm{C} 3 \mathrm{G}$, respectively $\left(\mathrm{a}^{\mathrm{N}} \approx 15.78 \mathrm{G}\right.$, and $\left.\mathrm{a}^{\mathrm{H}} \approx 2.73 \mathrm{G}\right)$.

extract $+\mathrm{Asc}+\mathrm{DMSO}+\mathrm{POBN}$ shows the signals of ${ }^{\circ} \mathrm{CH}_{3}$ indicating $\mathrm{HO}$ is formed from the system. The signals we see in 6) $\mathrm{C} 3 \mathrm{G}+\mathrm{Asc}+\mathrm{DMSO}+\mathrm{POBN}$ were stronger than those observed in 5) mainly due to the use of pure compound C3G. Additionally, the weaker signal observed in 5) could be attributed to the presence of other polyphenols in the blackberry extract capable of scavenging $\mathrm{HO}^{\circ}$. The generation of $\mathrm{HO}$ from the reaction of blackberry extract and ascorbic acid support our proposed mechanism. 


\subsection{Validation of mechanism using malvidin-3-O- $\beta$-glucoside}

In order to substantiate the hydroxylation mechanism in anthocyanin degradation, $\mathrm{M} 3 \mathrm{G}$ was used in a model system with Asc in the same ratios as $\mathrm{C} 3 \mathrm{G}$ previously. As shown in Fig. 8, M3G area declines rapidly with the addition of Asc, but remains stable in the control at ambient temperature. While monitoring at $510 \mathrm{~nm}$ for new colored peaks, one appeared quickly and increased in area until approximately 50 hours, followed by rapid decline. This mirrored the growth pattern of $6-\mathrm{OH}-\mathrm{C} 3 \mathrm{G}$, although that compound reached a maximum area

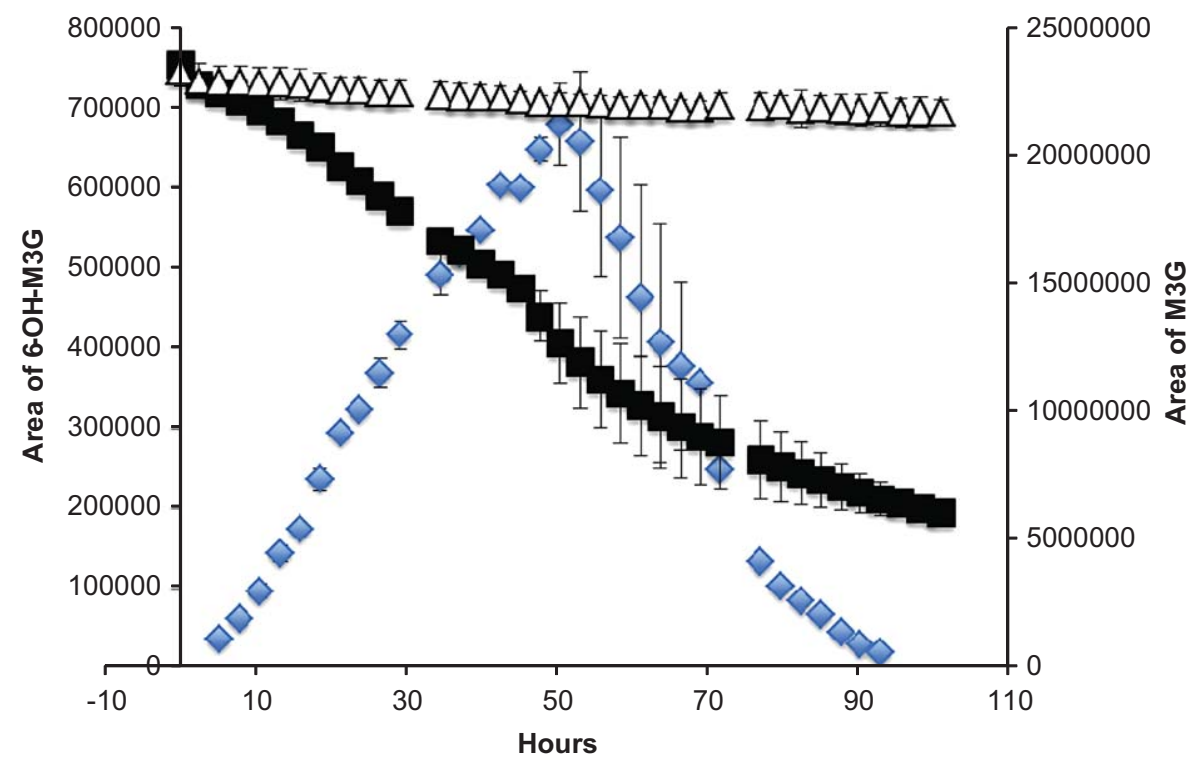

Fig. 8. Time course of M3G (with Asc $\mathbf{\square}$ ), M3G control ( $\Delta$ ), 6-hydroxy-M3G ( $\diamond)$. Bars represent standard error $(n=2)$.

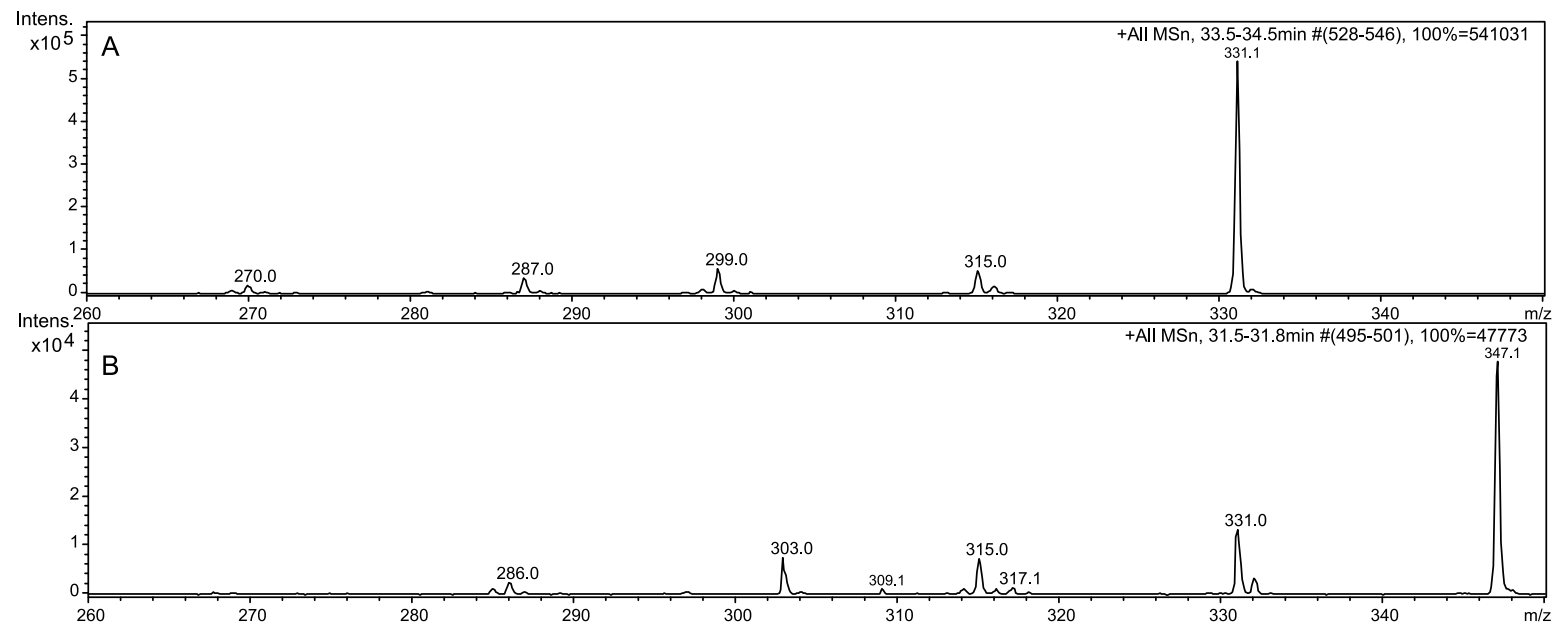

Fig. 9. LC-ESI-MS ${ }^{2}$ analysis of M3G (A), 6-hydroxy-malvidin (B). 
near 25 hours. The disparity could be explained by the relative stability differences of M3G to C3G. This peak represents a small percentage of the lost M3G (6.26\%) over 50 hours, similar to 6-OH-C3G accounting for $7.56 \%$ of the degraded C3G. Tandem mass spectrometry assisted in the tentative identification using a 16 amu difference for multiple peaks between M3G and the unidentified peak (Fig. 9). The 16 amu mass difference was identical to the $16 \mathrm{amu}$ differences between $\mathrm{C} 3 \mathrm{G}$ and 6-hydroxy-C3G leading to the identification of this peak as 6-hydroxy-malvidin-3-O- $\beta$-glucoside. This strengthens the hydroxylation radical mechanism for anthocyanins. Additional research is needed to determine if non-anthocyanin flavonoids are hydroxylated in the presence of Asc.

\section{Conclusions}

The addition of Asc led to an increased rate of degradation of $\mathrm{C} 3 \mathrm{G}$ in a model system based on the Asc:C3G ratio found in strawberries. The degradation products of $\mathrm{C} 3 \mathrm{G}$ were monitored for red colored compounds and the proposed structure of 6-hydroxy-cyanidin-3-O- $\beta$-glucoside was detected and described based on tandem mass spectrometric results. The role of hydroxyl radicals in formation of the novel compound was confirmed by ESR analysis. The peak area of the degradation product accounted for less than $8 \%$ of the area of $\mathrm{C} 3 \mathrm{G}$ degraded over time. Additional research is needed to identify other degradation products of anthocyanins catalyzed by ascorbic acid and to determine if hydroxylation of other polyphenols occurs via Asc catalyzed oxidation. The addition of food grade hydroxyl radical scavengers to berry juices may be a viable treatment to prevent ascorbic acid catalyzed degradation of anthocyanins.

\section{References}

[1] Pojer E, Mattivi F, Johnson D, Stockley CS. The case for anthocyanin consumption to promote human health: A review. Comp Rev Food Sci Food Safety. 2013;12:483-508.

[2] Patras A, Brunton NP, O'Donnell C, Tiwari BK. Effect of thermal processing on anthocyanin stability in foods; mechanisms and kinetics of degradation. Trends Food Sci \& Tech. 2010;21:3-11.

[3] Poei-Langston MS, Wrolstad RE. Color degradation in an ascorbic acid-anthocyanin-flavanol model system. J Food Sci. 1981;46(4):1218-22.

[4] Kader F, Irmouli M, Nicholas JP, Metche M. Involvement of blueberry peroxidase in the mechanisms of anthocyanin degradation of blueberry juice. J Food Sci. 2002;67(3):910-5.

[5] Li H, Guo A, Wang H. Mechanisms of oxidative browning of wine. Food Chem. 2008;108(1):1-13.

[6] Wilkes K, Howard LR, Brownmiller C, Prior RL. Changes in chokeberry (Aronia melanocarpa L.) polyphenols during juice processing and storage. J Agric Food Chem. 2014;62(18):4018-25.

[7] Tiwari BK, O’Donnell CP, Cullen PJ. Effect of non thermal processing technologies on the anthocyanin content of fruit juices. Trends Food Sci \& Tech. 2009;20(3):137-45.

[8] Jurd L. Some advances in the chemistry of anthocyanin-type plant pigments. In: Chichester CO. Ed. The chemistry of plant pigments. New York: Academic Press; 1972;123-42.

[9] Bradshaw MP, Barril C, Clark AC, Prenzler PD, Scollary GR. Ascorbic acid: A review of its chemistry and reactivity in relation to a wine environment. Crit Rev Food Sci Nutr. 2011;51(6):479-98.

[10] Brenes CH, Del Pozo-Insfran D, Talcott ST. Stability of copigmented anthocyanins and ascorbic acid in a grape juice model system. J Agric Food Chem. 2005;53(1):49-56.

[11] Garcia-Viguera C, Bridle P. Influence of structure on color stability of anthocyanins and flavylium salts with ascorbic acid. Food Chem. 1999;64(1):21-6.

[12] Iacobucci GA, Sweeny JG. The chemistry of anthocyanins and related flavylium salts. Tetrahedron. 1983;39(19):3005-38.

[13] Bakker J, Timberlake CF. Isolation, identification, and some characterization of new color-stable anthocyanins occurring in some red wines. J Agric Food Chem. 1997;45(1):35-43.

[14] Fulcrand H, Cameira dos Santos PJ, Sarni-Manchado P, Chaynier V, Favre-Bonvin J. Structure of new anthocyanin-derived wine pigments. J Chem Soc, Perkin Trans. 1(7):735-9. 
[15] He J, Santos-Buelga C, Mateus N, de Freitas V. Isolation and quantification of oligomeric pyranoanthocyanin-flavanol pigments from red wines by combination of column chromatographic techniques. J Chromato A. 2006;1134:215-25.

[16] Blanco-Vega D, Lopez-Bellido FJ, Alia-Robledo JM, Hermosin-Guiterrez I. HPLC-DAD-ESI-MS/MS characterization of pyranoanthocyanin pigments formed in model wine. J Agric Food Chem. 2011;59(17):9523-31.

[17] Rein MJ, Ollilainen V, Vahermo M, Yli-Kauhaluoma J, Heinonen M. Identification of novel pyranoanthocyanins in berry juices. Eur Food Res Technol. 2005;220(3):239-44.

[18] Hillebrand S, Schwarz M, Winterhalter P. Characterization of anthocyanins and pyranoanthocyanins from blood orange [Citrus sinensis (L.) Osbeck] juice. J Agric Food Chem. 2004;52(24):7331-8.

[19] Asenstorfer RE, Hayasaka Y, Jones GP. Isolation and structures of oligomeric wine pigments by bisulfite-mediated ion-exchange chromatography. J Agric Food Chem. 2001;49(12):5957-63.

[20] Monagas M, Gomez-Cordoves C, Bartolome B. Evolution of polyphenols in red wines from Vitis vinifera L. during aging in the bottle. Eur Food Res Technol. 2005;220(3):607-14.

[21] Somers TC. The polymeric nature of wine pigments. Phytochemistry. 1971;10(9):2175-86.

[22] Remy S, Fulcrand H, Labarbe B, Cheynier V, Moutounet M. First confirmation in red wine of product resulting from direct anthocyanintannin reactions. J Sci Food Agric. 2000;80(6):745-51.

[23] Reed JD, Krueger CG, Vestling MM. MALDI-TOF mass spectrometry of oligomeric food polyphenols. Phytochemistry. 2005;66(18):2248-63.

[24] Krueger CG, Vestling MM, Reed JD. Matrix-assisted laser desorption/ionization time-of-flight mass spectrometry of heteropolyflavan3-ols and glucosylated heteropolyflavans in sorghum [Sorghum bicolor (L.) Moench]. J Agric Food Chem. 2003;51(3):538-43.

[25] Howard LR, Prior RL, Liyanage R, Lay JO. Processing and storage effect on berry polyphenols: Challenges and implications for bioactive properties. J Agric Food Chem. 2012;60(27):6678-93.

[26] Hager TJ, Howard LR, Prior RL. Processing and storage effects on monomeric anthocyanins, percent polymeric color, and antioxidant capacity of processed blackberry products. J Agric Food Chem. 2008;56(3):689-95.

[27] Veberic R, Stampar F, Schmitzer V, Cunja V, Zupan A, Koron D, Mikulic-Petkocsek M. Changes in the contents of anthocyanins and other compounds in blackberry fruits due to freezing and long-term frozen storage. J Agric Food Chem. 2014;62(29):6926-35.

[28] Francis FJ. Food Colorants: Anthocyanins. Crit Rev Food Sci Nutr. 1989;28(4):273-314.

[29] United States Department of Agriculture - Agricultural Research Service. National nutrient database for standard reference release 27.

[30] Aaby K, Mazur S, Nes A, Skrede G. Phenolic compounds in strawberry (Fragaria x ananassa Duch.) fruits: Composition in 27 cultivars and changes during ripening. Food Chem. 2012;132(1):96-7.

[31] Giusti MM, Rodriguez-Saona LE, Griffin D, Wrolstad RE. Electrospray and tandem mass spectrometry as tools for anthocyanin characterization. J Agric Food Chem. 1999;47(11):4657-64.

[32] Kelm MA, Johnson JC, Robbins RJ, Hammerstone JF, Schmitz HH. High-performance liquid chromatography separation and purification of cacao (Theobroma cacao L.) procyanidins according to degree of polymerization using diol stationary phase. J Agric Food Chem. 2006;54(5):1571-6.

[33] Saito N, Yokoi M, Yamaji M, Honda T. Anthocyanidin glycosides from the flowers of Alstroemeria. Phytochemistry. 1985;24(9):21256.

[34] Tatsuzawa F, Saito N, Murata N, Shinoda K, Shigihara A, Honda T. Two novel 6-hydroxyanthocyanins in the flowers of Alstroemeria 'Westland.' Heterocycles. 2002;57(10):1787-92.

[35] Talcott ST, Brenes CH, Pires DM, Del Pozo-Insfran D. Phytochemical stability and color retention of copigmented and processed muscadine grape juice. J Agric Food Chem. 2003;51(4):957-63.

[36] Davidek J, Velisek J, Pokorny J. Chemical changes during food processing. Amsterdam: Elsevier 1990. p. 448.

[37] Taqui Khan MM, Martell AE. Metal ion and metal chelate catalyzed oxidation of ascorbic acid by molecular oxygen. I. Cupric and ferric ion catalyzed oxidation. J Am Chem Soc. 1967;89(16):4176-85.

[38] Zhao MJ, Jung L. Kinetics of the competitive degradation of deoxyribose and other molecules by hydroxyl radicals produced by the fenton reaction in the presence of ascorbic acid. Free Rad Res. 1995;23(3):229-43.

[39] Graf E, Mahoney JR, Bryant RG, Eaton JW. Iron-catalyzed hydroxyl radical formation. Stringent requirement for free iron coordination site. J Biol Chem. 1984;259(6):3620-24.

[40] Mahoney JR, Graf E. Role of alpha-tocopherol, ascorbic acid, citric acid and EDTA as oxidants in model systems. J Food Sci. 1986;51(5):1293-6.

[41] Martell AE. Chelates of ascorbic acid: Formation and catalytic properties. In: Ascorbic acid: Chemistry, metabolism, and uses. 1982;pp. 153-78. DOI:10.1021/ba-1982-0200.ch007 
[42] Ozkan M, Yemenicioglu A, Asefi N, Cemeroglu B. Degradation kinetics of anthocyanins from sour cherry, pomegranate, and strawberry juices by hydrogen peroxide. Food Chem Toxic. 2002;67(2):525-9.

[43] Iversen CK. Black currant nectar: Effect of processing and storage on anthocyanin and ascorbic acid content. J Food Sci. 1999;64(1):3741.

[44] Lopez-Serrano M, Ros Barcelo A. $\mathrm{H}_{2} \mathrm{O}_{2}$-mediated pigment decay in strawberry as a model system for studying color alterations in processed plant foods. J Agric Food Chem. 1999;47(3):824-7.

[45] Sondheimer E, Kertesz ZI. The kinetics of the oxidation of strawberry anthocyanin by hydrogen peroxide. Food Res. 1952;17(1):28898.

[46] Tsuda T, Ohshima K, Kawakishi S, Osawa T. Oxidation products of cyanidin-3-O- $\beta$-glucoside with a free radical initiator. Lipids. 1996;31(12):1259-63.

[47] Kiliç M, Koçtürk G, Nevim S, Çinar Z. A model for prediction of product distributions for the reactions of phenol derivatives with hydroxyl radicals. Chemosphere. 2007;69(9):1396-408.

[48] Qian SY, Kadiiska MB, Guo Q, Mason RP. A novel protocol to identify and quantify all spin trapped free radicals from in vitro/in vivo interaction of $\mathrm{HO}^{\circ}$ and DMSO: LC/ESR, LC/MS, and dual spin trapping combinations. Free Rad Biol Med. 2005;38(1):125-35.

[49] Zhong Z, Connor HD, Li X, Mason RP, Forman DT, Lemasters JJ, Thurman RG. Reduction of ciclosporin and tacrolimus nephrotoxicity by plant polyphenols. J Pharm Pharmacol. 2006;58(11):1533-43.

[50] Qian SY, Buettner GR. Iron and dioxygen chemistry is an important route to initiation of biological free radical oxidations: An electron paramagnetic resonance spin trapping study. Free Radic Biol Med. 1999;26(11-12):1447-56. 